\title{
GMR
}

\section{Association of LRP5 Ala1330Val polymorphism with fracture risk: a meta-analysis}

\author{
J. Peng, Y. Lu, Y.J. Liu and Y.L. Zhan \\ Department of Orthopedics, \\ Shanghai Jiaotong University Affiliated Sixth People's Hospital, Shanghai, China \\ Corresponding author: Y.L. Zhan \\ E-mail: zhanyulin03@sina.com
}

Genet. Mol. Res. 15 (1): gmr.15017552

Received August 31, 2015

Accepted November 13, 2015

Published March 31, 2016

DOI http://dx.doi.org/10.4238/gmr.15017552

\begin{abstract}
Numerous studies have evaluated the association between the Ala1330Val polymorphism of the low-density lipoprotein receptor-related proteins 5 (LRP5) gene and fracture risk. However, the specific association is still controversial. The aim of this study was to investigate their correlation via metaanalysis. and EMBASE databases were searched, and data were extracted independently by two reviewers. Odds ratios (ORs) with corresponding 95\% confidence intervals (Cls) were used to assess the strength of the associations. Statistical analysis was performed using the STATA 12.0 software. Seven studies, involving 808 cases and 1586 controls, were included in the analysis. Meta-analysis results showed no significant association between the LRP5 Ala1330Val polymorphism and fracture risk (ValVal vs AlaAla: OR $=1.25$, $95 \% \mathrm{Cl}=0.82-1.91 ;$ AlaVal vs AlaAla: $\mathrm{OR}=1.16,95 \% \mathrm{Cl}=0.95-1.42$; dominant model: $\mathrm{OR}=1.77,95 \% \mathrm{Cl}=0.96-1.41$; recessive model: $\mathrm{OR}=1.21,95 \% \mathrm{Cl}$ $=0.80-1.83$ ). Taking into account the effect of ethnicity, the LRP5 Ala1330Val polymorphism was not associated with the risk of fracture in Asians or Caucasians. The results of the current meta-analysis indicate that the LRP5 Ala1330Val polymorphism may not be correlated with fracture susceptibility.
\end{abstract}

Key words: LRP5; Ala1330Val; Gene; Fracture risk; Meta-analysis 


\section{INTRODUCTION}

Fractures are an increasingly common cause of morbidity, affecting approximately 78-94 of 100,000 people, and therefore, they cause a serious burden to health services worldwide (Cauley et al., 2008; Lanza et al., 2013). Prevention of fractures using drugs could potentially be as expensive as medical treatment of fractures. However, the identification of individuals at high-risk of disabling fractures would allow careful allocation of these expensive treatments to the most at-risk individuals. Numerous studies have attempted to explore the pathogenesis of fractures. Their occurrence is influenced by many environmental factors, such as older age, low heel quantitative ultrasound stiffness in$\operatorname{dex}(<78 \%)$, history of fracture, recent falls, and failing of the chair test (Deng et al., 2015). In addition, genetic factors also play a key role in the pathogenesis of fractures (Oei et al., 2014; Gao et al., 2015).

The Wnt signaling pathway, including the low-density lipoprotein receptor-related protein 5 (LRP5) and Wnt proteins, is a vitally important pathway that regulates osteoblastic activity and bone mass (Gong et al., 2001). LRP5 is crucial for normal bone morphology, development, mechanosensation, and bone formation (Li et al., 2005). In osteoblasts, LRP5 can transduce canonical signals to promote the renewal of stem cells, the stimulation of pre-osteoblast replication, the induction of osteoblastogenesis, and the inhibition of osteoblast and osteocyte apoptosis by increasing the levels of $\beta$-catenin and altering gene expression through the Lef/Tcf transcription factors (Krishnan et al., 2006). The human LRP5 gene is located on chromosome 11q13.4, which consists of 22 introns and 23 exons and spans approximately $160 \mathrm{~kb}$ (Smith et al., 2005). Recently, a number of polymorphisms have been described in the LRP5 gene (http://www.ncbi.nlm.nih.gov/SNP). Among them, the Ala1330Val (rs3736228, exon 18) polymorphism has been studied most extensively (van Meurs et al., 2008) and was found to affect bone mineral density (Sassi et al., 2014).

To date, several reports have addressed the association between the LRP5 Ala1330Val polymorphism and fracture. However, the conclusions have been conflicting. Some studies have a limited sample size and subsequently suffer from low detection power (Zhang et al., 2013). Therefore, we performed a meta-analysis to clarify the association between the LRP5 Ala1330Val polymorphism and fracture risk.

\section{MATERIAL AND METHODS}

\section{Search strategies}

A comprehensive search was carried out using and EMBASE databases to identify casecontrol studies that were published prior to August 28, 2015, and that examined the association of the LRP5 Ala1330Val polymorphism with fracture risk. Searches were carried out using the following terms: "low-density lipoprotein receptor-related proteins 5/LRP5", "fracture", "polymorphism", "single nucleotide polymorphism", and "genetic polymorphism", without posing restrictions on publication language. The references cited in the identified articles were searched manually to find additional studies. If the information provided in the literature was not sufficiently clear, the original authors were contacted for additional raw data. When overlapping data existed, only the study with more complete information was adopted.

\section{Inclusion and exclusion criteria}

Inclusion and exclusion criteria were established before searching the literature. To be 
included in our meta-analysis, studies had to 1) evaluate the association between the LRP5 Ala1330Val polymorphism and fracture risk, 2) be designed as case-control studies, and 3) provide sufficient data to estimate an odd ratio (OR) with its $95 \%$ confidence interval $(95 \% \mathrm{Cl})$. The following publications were excluded: 1) duplicate data, 2) reviews, 3) studies not relevant to fracture or the specific polymorphism, 4) animal experiments, 5) non-case-control designs, or 6) unavailability of raw data even after contacting the author.

\section{Data extraction}

Two investigators (J. Peng and L. Yu) extracted data independently and reached consensus on the following characteristics of the selected studies: first author's name, the year of publication, ethnicity of the study population, number of participants, genotype distribution and control source, and the allele and evidence of Hardy-Weinberg equilibrium (HWE), all of which are listed in Table 1.

\section{Statistical analysis}

All statistical analyses were performed using the Stata software (Version 12.0; StataCorp., College Station, TX, USA). ORs and $95 \% \mathrm{Cls}$ were calculated to assess the strength of association between the LRP5 Ala1330Val polymorphism and fracture risk for 4 genetic models: a homozygote comparison (ValVal vs AlaAla), a heterozygote comparison (AlaVal vs AlaAla), a dominant model (ValVal+AlaVal vs AlaAla), and a recessive model (AlaAla+AlaVal vs ValVal). In addition, we also performed stratification analyses by race. The heterogeneity among the studies was evaluated by the $\mathrm{I}^{2}$ test ranging from 0 to $100 \%$ to describe the percentage of between-study variation caused by heterogeneity. If heterogeneity was found among the studies, the pooled OR was estimated by the fixed-effect model $\left(I^{2}<50 \%\right)$. Otherwise, the random-effect model was used to estimate the pooled OR. The HWE of the genotype frequencies of the controls was assessed via the $\chi^{2}$ test. Sensitivity analysis was performed by sequential omission of non-HWE studies. Publication bias was investigated using a Begg's funnel plot.

\section{RESULTS}

\section{Study characteristics}

The study selection flow chart is shown in Figure 1. In total, 110 papers were retrieved by the literature search. Seven full-text papers were included in this meta-analysis and 103 studies were excluded (Mizuguchi et al., 2004; Ferrari et al., 2005; Hartikka et al., 2005; Korvala et al., 2010; Liu et al., 2010; Saarinen et al., 2010; Xuan et al., 2014). Based on the 7 selected studies, 808 cases and 1586 controls were included in the pooled analysis. All study characteristics are summarized in Table 1. All the studies were published in English. The HWE test was performed on genotype distribution of the controls, and all of them were in agreement with HWE, except Saarinen et al. (2010). There were 3 studies conducted on Asian populations (Mizuguchi et al., 2004; Liu et al., 2010; Xuan et al., 2014) and 4 studies on Caucasian populations (Ferrari et al., 2005; Hartikka et al., 2005; Korvala et al., 2010; Saarinen et al., 2010). 


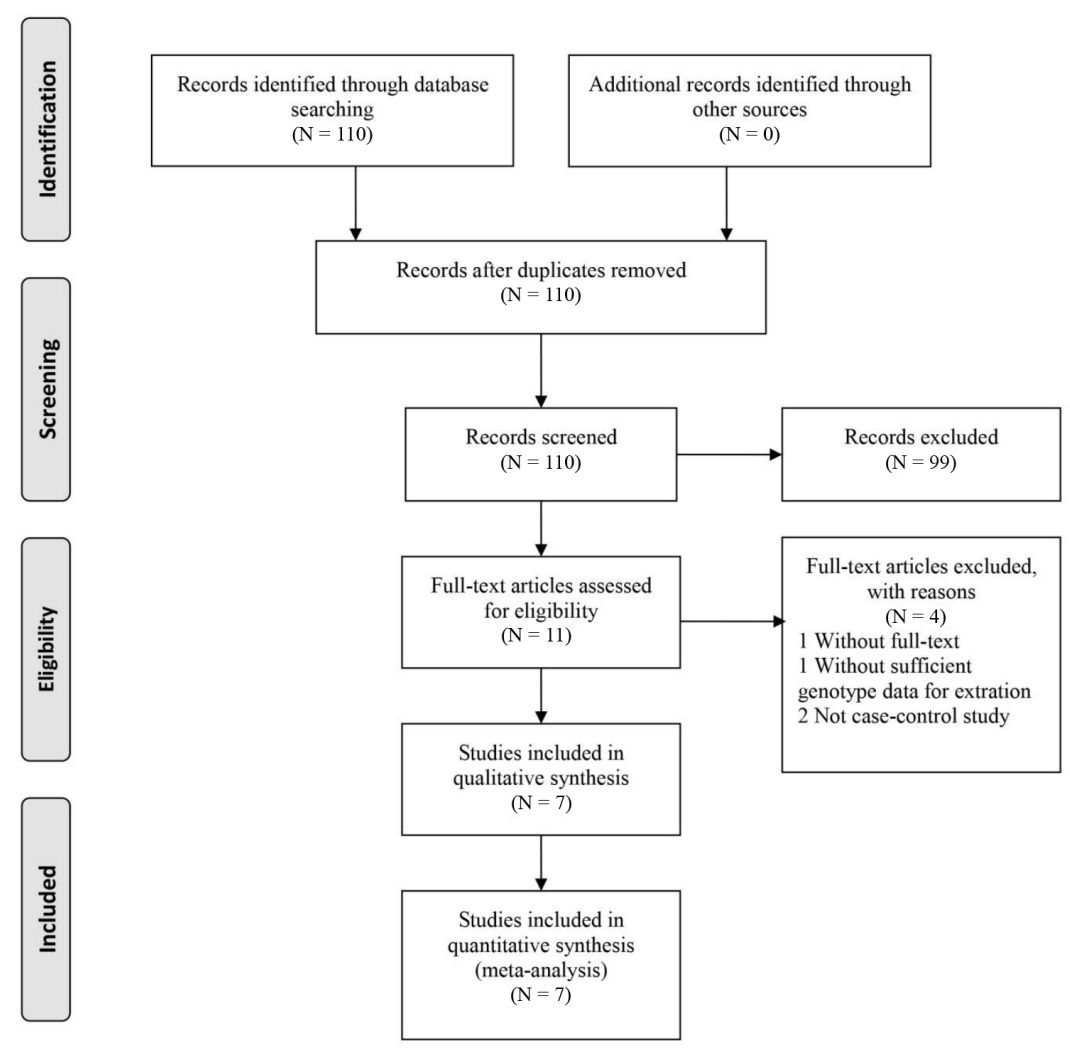

Figure 1. Detailed process of study selection.

\section{Table 1. Characteristics of the included studies for meta-analysis.}

\begin{tabular}{l|l|l|c|c|c|c|c|c|c|c|c}
\hline Study & Year & Area & Race & Cases/Controls & \multicolumn{2}{|c|}{ Genotypes for cases } & \multicolumn{2}{c|}{ Genotypes for controls } & HWE test \\
\hline & & & & & AlaAla & AlaVal & ValVal & AlaAla & AlaVal & ValVal & \\
\hline Mizuguchi & 2004 & Japan & Asians & $126 / 131$ & 62 & 53 & 11 & 76 & 48 & 7 & 0.87 \\
\hline Hartikka & 2005 & Finland & Caucasians & $20 / 88$ & 13 & 6 & 1 & 50 & 33 & 5 & 0.88 \\
\hline Ferrari & 2005 & Switzerland & Caucasians & $78 / 86$ & 43 & 33 & 2 & 61 & 23 & 2 & 0.92 \\
\hline Saarinen & 2010 & Finland & Caucasians & $66 / 235$ & 53 & 6 & 7 & 197 & 18 & 20 & 0.00 \\
\hline Korvala & 2010 & Finland & Caucasians & $144 / 240$ & 124 & 19 & 1 & 217 & 22 & 1 & 0.59 \\
\hline Liu & 2010 & China & Asians & $284 / 728$ & 171 & 99 & 14 & 450 & 245 & 33 & 0.96 \\
\hline Xuan & 2014 & China & Asians & $90 / 78$ & 64 & 23 & 3 & 50 & 25 & 3 & 0.95 \\
\hline
\end{tabular}

HWE = Hardy-Weinberg equilibrium.

\section{Meta-analysis}

Table 2 lists the main results of the pooled analysis and Figure 2 shows the association of the LRP5 Ala1330Val polymorphism with fracture risk in the form of forest plots. Overall, the metaanalysis results identified no significant association between the LRP5 Ala1330Val polymorphism and susceptibility to fracture (ValVal vs AlaAla: $\mathrm{OR}=1.25,95 \% \mathrm{Cl}=0.82-1.91$; AlaVal vs AlaAla: OR $=1.16,95 \% \mathrm{Cl}=0.95-1.42$; dominant model: $\mathrm{OR}=1.77,95 \% \mathrm{Cl}=0.96-1.41$; recessive model: OR $=1.21,95 \% \mathrm{Cl}=0.80-1.83)$. In order to obtain the exact consequence of the relationship between 
LRP5 Ala1330Val polymorphism and fracture susceptibility, we performed analyses stratified by ethnicity. We detected an insignificant association in both Asians (ValVal vs AlaAla: OR $=1.25$, $95 \% \mathrm{Cl}=0.75-2.09 ;$ AlaVal vs AlaAla: $\mathrm{OR}=1.07,95 \% \mathrm{Cl}=0.84-1.35$; dominant model: $\mathrm{OR}=1.08$, $95 \% \mathrm{Cl}=0.86-1.36$; recessive model: $\mathrm{OR}=1.21,95 \% \mathrm{Cl}=0.73-2.00)$ and Caucasians (ValVal vs AlaAla: $\mathrm{OR}=1.26,95 \% \mathrm{Cl}=0.60-2.65 ;$ AlaVal vs AlaAla: $\mathrm{OR}=1.46,95 \% \mathrm{Cl}=0.99-2.14$; dominant model: $\mathrm{OR}=1.39,95 \% \mathrm{Cl}=0.98-1.98$; recessive model: $\mathrm{OR}=1.22,95 \% \mathrm{Cl}=0.58-2.55)$.

\section{Sensitivity analysis}

We conducted a sensitivity analysis by omission of one non-HWE study (Saarinen et al., 2010) to evaluate the stability of the meta-analysis. The result was not significantly altered, indicating that the data in this meta-analysis are relatively stable and credible. The detailed data are presented in Table 2.

Table 2. Summary ORs and $95 \% \mathrm{Cl}$ of the association between the LRP5 Ala1330Val polymorphism and fracture risk.

\begin{tabular}{|c|c|c|c|c|c|c|c|c|}
\hline \multirow[t]{2}{*}{ Subgroup } & \multirow[t]{2}{*}{ Genetic model } & \multicolumn{2}{|c|}{ Sample size } & \multirow[t]{2}{*}{ Model type } & \multicolumn{2}{|c|}{ Heterogeneity test } & \multicolumn{2}{|c|}{ Association test } \\
\hline & & Cases & Controls & & $\mathrm{I}^{2}$ & $\mathrm{P}$ & OR & $95 \% \mathrm{Cl}$ \\
\hline \multirow[t]{4}{*}{ Overall } & ValVal vs AlaAla & 808 & 1586 & Fixed & $0.0 \%$ & 0.97 & 1.25 & $0.82-1.91$ \\
\hline & AlaVal vs AlaAla & & & Fixed & $13.3 \%$ & 0.33 & 1.16 & $0.95-1.42$ \\
\hline & Dominant model & & & Fixed & $14.2 \%$ & 0.32 & 1.17 & $0.96-1.41$ \\
\hline & Recessive model & & & Fixed & $0.0 \%$ & 0.99 & 1.21 & $0.80-1.83$ \\
\hline \multirow[t]{4}{*}{ Caucasians } & ValVal vs AlaAla & 308 & 649 & Fixed & $0.0 \%$ & 0.97 & 1.26 & $0.60-2.65$ \\
\hline & AlaVal vs AlaAla & & & Fixed & $0.0 \%$ & 0.40 & 1.46 & $0.99-2.14$ \\
\hline & Dominant model & & & Fixed & $0.0 \%$ & 0.40 & 1.39 & $0.98-1.98$ \\
\hline & Recessive model & & & Fixed & $0.0 \%$ & 0.98 & 1.22 & $0.58-2.55$ \\
\hline \multirow[t]{4}{*}{ Asians } & ValVal vs AlaAla & 500 & 937 & Fixed & $0.0 \%$ & 0.57 & 1.25 & $0.75-2.09$ \\
\hline & AlaVal vs AlaAla & & & Fixed & $6.2 \%$ & 0.35 & 1.07 & $0.84-1.35$ \\
\hline & Dominant model & & & Fixed & $25.1 \%$ & 0.26 & 1.08 & $0.86-1.36$ \\
\hline & Recessive model & & & Fixed & $0.0 \%$ & 0.70 & 1.21 & $0.73-2.00$ \\
\hline Consistent with & ValVal vs AlaAla & 742 & 1351 & Fixed & $0.0 \%$ & 0.93 & 1.24 & $0.77-2.00$ \\
\hline \multirow[t]{3}{*}{ HWE } & AlaVal vs AlaAla & & & Fixed & $27.5 \%$ & 0.23 & 1.16 & $0.94-1.42$ \\
\hline & Dominant model & & & Fixed & $27.9 \%$ & 0.23 & 1.16 & $0.95-1.41$ \\
\hline & Recessive model & & & Fixed & $0.0 \%$ & 0.97 & 1.19 & $0.75-1.91$ \\
\hline
\end{tabular}

\section{Publication bias}

We analyzed the Begg's funnel plot to assess the publication bias. The shape of the funnel plot did not reveal any evidence of obvious asymmetry in all comparisons in the overall population, which implies that the publication bias was low in the present meta-analysis (Table 2 and Figure 3 ).

\section{DISCUSSION}

In the past decade, fractures are one of the most frequent diseases worldwide. Previous studies have demonstrated that fracture has a complex etiology, and is considered a multifactorial polygenic disease. LRP5 is a transmembrane protein that functions as a coreceptor in the Wnt-signaling pathway and modulates bone mass accrual (Gong et al., 2001). Ala1330Val in exon 18 of LRP5 is a missense polymorphism and recent studies found an association between this polymorphism and fracture risk. However, the published results have been inconsistent, possibly due to differences in study design, study populations, and sample sizes. Via meta-analysis, these disadvantages can be resolved through the systematic 


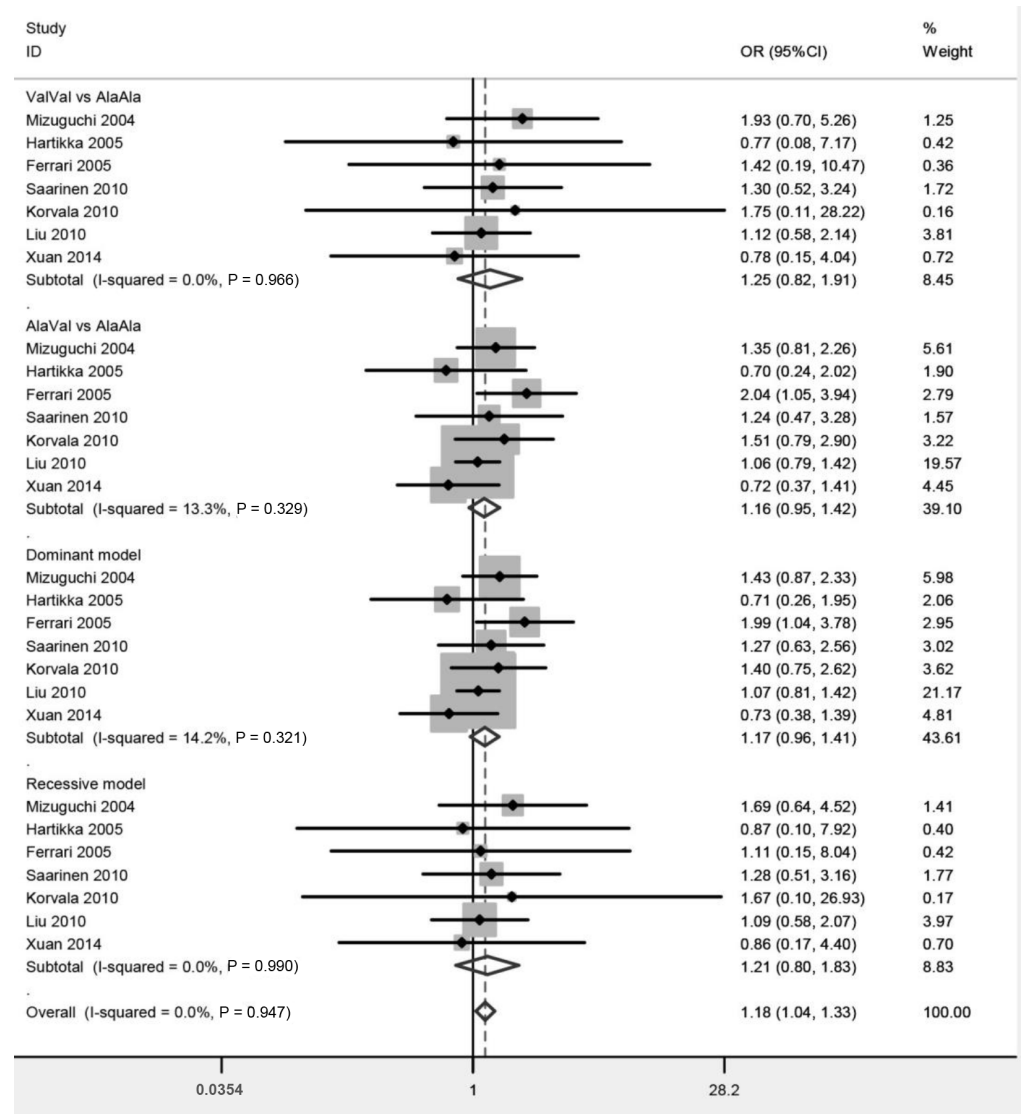

Figure 2. Meta-analysis of the relationship between the LRP5 Ala1330Val polymorphism and fracture risk.

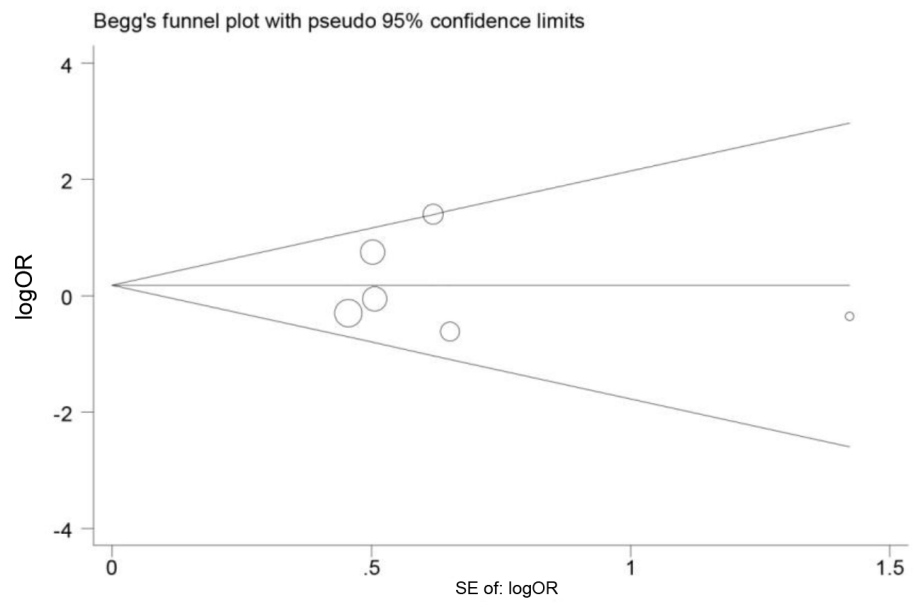

Figure 3. Begg's funnel plot test of publication bias. 
collection of the available data, thus increasing the statistical power and enhancing the precision of the results (Thakkinstian et al., 2004).

Our meta-analysis quantitatively assessed the relation between the LRP5 Ala1330Val polymorphism and fracture risk. Seven case-control studies were included and assessed, encompassing a total of 808 essential fracture patients and 1586 healthy controls. Overall, no significant association between fracture risk and the LRP5 Ala1330Val polymorphism could be identified when all studies were pooled into the meta-analysis. In the stratified analysis by ethnicity, we found no relation between fracture risk and the LRP5 Ala1330Val polymorphism in either Caucasians or Asians. It is possible that the observed rare variants may contribute to fractures in association with other unfavorable environmental and genetic factors. Previous studies found that the LRP5 haplotype (E644E, V667M, V1119V, and A1330V), in combination with the vitamin D receptor haplotype, was associated with stress fractures (Korvala et al., 2010).

This study has several potential limitations. First, the number of included studies was not sufficiently large for a comprehensive analysis, and some included studies of small size might not have had enough statistical power. Second, publication bias may have occurred, although no obvious bias was observed in the bias diagnostics. Additionally, the effect of potential gene-gene and gene-environment interactions could not be addressed in this meta-analysis.

In summary, our meta-analysis suggests that the LRP5 Ala1330Val polymorphism is not associated with fracture risk. However, more prospective studies with a larger sample size are still required to confirm these findings.

\section{Conflicts of interest}

The authors declare no conflict of interest.

\section{REFERENCES}

Cauley JA, Wampler NS, Barnhart JM, Wu L, et al.; Women's Health Initiative Observational Study (2008). Incidence of fractures compared to cardiovascular disease and breast cancer: the Women's Health Initiative Observational Study. Osteoporos. Int. 19: 1717-1723.http://dx.doi.org/10.1007/s00198-008-0634-y

Deng W, Han JC, Chen L and Qi WL (2015). Estrogen receptor alpha gene Pvull polymorphism and risk of fracture in postmenopausal women: a meta-analysis. Genet. Mol. Res. 14: 1293-1300.http://dx.doi.org/10.4238/2015.February.13.8

Ferrari SL, Deutsch S, Baudoin C, Cohen-Solal M, et al. (2005). LRP5 gene polymorphisms and idiopathic osteoporosis in men. Bone 37: 770-775.http://dx.doi.org/10.1016/j.bone.2005.06.017

Gao J, Wang L and Zhu J (2015). Influence of Bsml polymorphism in vitamin D receptor gene on the risk of fracture in Caucasian populations: a meta analysis. Int. J. Clin. Exp. Med. 8: 589-597.

Gong Y, Slee RB, Fukai N, Rawadi G, et al.; Osteoporosis-Pseudoglioma Syndrome Collaborative Group (2001). LDL receptorrelated protein 5 (LRP5) affects bone accrual and eye development. Cell 107: 513-523.http://dx.doi.org/10.1016/S00928674(01)00571-2

Hartikka H, Mäkitie O, Männikkö M, Doria AS, et al. (2005). Heterozygous mutations in the LDL receptor-related protein 5 (LRP5) gene are associated with primary osteoporosis in children. J. Bone Miner. Res. 20: 783-789.http://dx.doi. org/10.1359/JBMR.050101

Korvala J, Hartikka H, Pihlajamäki H, Solovieva S, et al. (2010). Genetic predisposition for femoral neck stress fractures in military conscripts. BMC Genet. 11: 95.http://dx.doi.org/10.1186/1471-2156-11-95

Krishnan V, Bryant HU and Macdougald OA (2006). Regulation of bone mass by Wnt signaling. J. Clin. Invest. 116: $1202-1209$. http://dx.doi.org/10.1172/JCl28551

Lanza LL, McQuay LJ, Rothman KJ, Bone HG, et al. (2013). Use of depot medroxyprogesterone acetate contraception and incidence of bone fracture. Obstet. Gynecol. 121: 593-600.http://dx.doi.org/10.1097/AOG.0b013e318283d1a1

Li X, Zhang Y, Kang H, Liu W, et al. (2005). Sclerostin binds to LRP5/6 and antagonizes canonical Wnt signaling. J. Biol. Chem. 280: 19883-19887.http://dx.doi.org/10.1074/jbc.M413274200 
Liu JM, Zhang MJ, Zhao L, Cui B, et al. (2010). Analysis of recently identified osteoporosis susceptibility genes in Han Chinese women. J. Clin. Endocrinol. Metab. 95: E112-E120.http://dx.doi.org/10.1210/jc.2009-2768

Mizuguchi T, Furuta I, Watanabe Y, Tsukamoto K, et al. (2004). LRP5, low-density-lipoprotein-receptor-related protein 5, is a determinant for bone mineral density. J. Hum. Genet. 49: 80-86.http://dx.doi.org/10.1007/s10038-003-0111-6

Oei L, Hsu YH, Styrkarsdottir U, Eussen BH, et al. (2014). A genome-wide copy number association study of osteoporotic fractures points to the 6p25.1 locus. J. Med. Genet. 51: 122-131.http://dx.doi.org/10.1136/imedgenet-2013-102064

Saarinen A, Mäyränpää MK, Lehesjoki AE and Mäkitie O (2010). Low-density lipoprotein receptor-related protein 5 (LRP5) variation in fracture prone children. Bone 46: 940-945.http://dx.doi.org/10.1016/j.bone.2009.12.022

Sassi R, Sahli H, Souissi C, El Mahmoudi H, et al. (2014). Association of LRP5 genotypes with osteoporosis in Tunisian postmenopausal women. BMC Musculoskelet. Disord. 15: 144.http://dx.doi.org/10.1186/1471-2474-15-144

Smith AJ, Gidley J, Sandy JR, Perry MJ, et al. (2005). Haplotypes of the low-density lipoprotein receptor-related protein 5 (LRP5) gene: are they a risk factor in osteoarthritis? Osteoarthritis Cartilage 13: 608-613.http://dx.doi.org/10.1016/j. joca.2005.01.008

Thakkinstian A, D'Este C, Eisman J, Nguyen T, et al. (2004). Meta-analysis of molecular association studies: vitamin D receptor gene polymorphisms and BMD as a case study. J. Bone Miner. Res. 19: 419-428.http://dx.doi.org/10.1359/ JBMR.0301265

van Meurs JB, Trikalinos TA, Ralston SH, Balcells S, et al.; GENOMOS Study (2008). Large-scale analysis of association between LRP5 and LRP6 variants and osteoporosis. JAMA 299: 1277-1290.http://dx.doi.org/10.1001/jama.299.11.1277

Xuan M, Wang Y, Wang W, Yang J, et al. (2014). Association of LRP5 gene polymorphism with type 2 diabetes mellitus and osteoporosis in postmenopausal women. Int. J. Clin. Exp. Med. 7: 247-254.

Zhang H, Ma H, Xu Y and Li L (2013). Association of SMAD7 rs12953717 polymorphism with cancer: a meta-analysis. PLoS One 8: e58170.http://dx.doi.org/10.1371/journal.pone.0058170 